\title{
Ventricular repolarization indexes in patients treated with hydroxychloroquine - azithromycin combination for COVID-19
}

\author{
Gunay $\mathrm{S}^{1}$, Caliskan $\mathrm{S}^{2}$, Sigirli $\mathrm{D}^{3}$, Sahin $\mathrm{E}^{4}$ \\ Bursa Uludag University Faculty of Medicine, Department of Cardiology, Bursa, Turkey. \\ seydagunaymedical@yahoo.com
}

\begin{abstract}
BACKGROUND: Combination of hydroxychloroquine and azithromycin for the treatment of coronavirus disease 2019 (COVID-19) carries increased risk of corrected QT (QTc) prolongation and cardiac arrhythmias. OBJECTIVE: To characterize the ventricular repolarization indexes which are associated with malignant ventricular arrhythmias in patients treated with hydroxychloroquine and concomitant azithromycin for COVID-19. METHOD: A total of 81 patients who had hydroxychloroquine and azithromycin combination therapy because of possible or reverse-transcription polymertase chain reaction (RT-PCR) confirmed diagnosis of COVID-19 were included in the study. Baseline and control electrocardiograms (before and after treatment) were analyzed retrospectively. Tp-e interval, Tp-e/QT and Tp-e/QTc ratios, which are ventricular repolarization indexes, were calculated.

RESULTS: While there was no significant increase in QTc interval in patients receiving combination therapy, there was a significant increase in ventricular repolarization indexes.

CONCLUSION: The increase in ventricular replarization indexes is associated with the risk of arrhythmia. In patients using QTc prolonging medication for COVID-19 treatment, QTC monitoring alone may not be sufficient to follow-up for arrhythmia. Even if there is no prolongation in QTc, an increase in ventricular repolarization indexes may be seen (Tab. 5, Ref. 37). Text in PDF www.elis.sk KEY WORDS: hydroxychloroquine, azithromycin, QTc prolongation, Tp-e, repolarization, arrhythmia.
\end{abstract}

\section{Introduction}

All over the world, more than 2 million individuals have been infected with the coronavirus 2019 disease (COVID-19) and almost 500 million people worldwide are predicted to be infected (1). As of April 2020, the mortality rates in different countries were between $1 \%$ and $13 \%(2)$. In Turkey, it was $2.64 \%$.

Unfourtunately, an effective medical therapy for COVID-19 is not yet available. Hence, researches on potential therapeutic options were prompted because of the emergence of COVID-19. Hydroxychloroquine, chloroquine, azithromycin, lopinavir/ritonavir, remdesivir, ribavirin are some of the earlier candidates (3).

Hydroxychloroquine has effects against SARS-CoV2 in vitro and exhibits a less toxic profile than chloroquine (4). This drug is well known and has been used to treat autoimmune diseases for a long time. Azithromycin is a macrolide and is effective against Zika and up-regulates interferons I and III (5-7).

Although there is no approved drug to prevent or treat SARS$\mathrm{CoV}-2$ infection, Gutret et al. reported that the combination of

'Bursa Uludag University Faculty of Medicine, Department of Cardiology, Bursa, Turkey, ${ }^{2}$ Istanbul Bahcelievler State Hospital, Department of Cardiology, Istanbul, Turkey, ${ }^{3}$ Bursa Uludag University Faculty of Medicine, Department of Biostatistics, Bursa, Turkey, and ${ }^{4}$ Istanbul Bahcelievler State Hospital, Department of Internal Medicine, Istanbul, Turkey

Address for correspondence: S. Gunay, MD, Uludag University, Faculty of Medicine, Department of Cardiology, Görükle 16059, Bursa, Turkey Phone: 0505.278.0777, Fax: 0224.2951628 hydroxychloroquine with azithromycin (HQ/AZ) may improve clinical outcomes and viral load of infected patients (8). Both hydroxychloroquine and azithromycin may lead to QT prolongation-related life-threatening arrhythmias through the blockage of potassium channels. Their combination seems to be more harmful than their single use (9). In addition, because of concomitant cardiac injury from SARS-CoV-2, safe drugs may even cause advers events (10). Across the world many centers use this combination even if there is a lack of evidence in terms of effectiveness (11).

Although the patients treated with QTc prolonging drugs are followed up with corrected QT interval ( QTc), QTc may not be enough to detect arrhythmia risk as early as possible. Electrocardiographic measurements other than QTc are also available to assess ventricular repolarization. On an electrocardiogram (ECG), the interval from the peak to the end of T wave (Tpe) may represent the dispersion of ventricular repolarization. Malignant ventricular arrhythmias are associated with Tpe interval and Tpe/ QT ratio.

In this study, we aimed to evaluate the ventricular arrhytmia risk in patients treated with hydroxychloroquine and azithromycin combination for COVID-19 by using ventricular repolarization indexes.

\section{Method}

In this study, we included adult patients who were treated with $\mathrm{HQ} / \mathrm{AZ}$ combination therapy for possible or reverse-transcription 
Tab. 1. Risk score for drug-associated QTc prolongation (12).

\begin{tabular}{lc}
\hline Risk Factors & Points \\
\hline Age $\geq 68$ years & 1 \\
Female sex & 1 \\
Loop diuretic & 1 \\
Serum potassium $\leq 3.5 \mathrm{mEq} / \mathrm{L}$ & 2 \\
Admission QTc $\geq 450 \mathrm{~ms}$ & 2 \\
Acute myocardial infarction & 2 \\
$\geq 2$ QTc-prolonging drugs & 3 \\
Sepsis & 3 \\
Heart failure & 3 \\
One QTc-prolonging drug & 3 \\
Maximum Risk Score & 21 \\
\hline
\end{tabular}

A Tisdale score of $\leq 6$ predicts low risk, 7-10 medium risk, and $\geq 11$ high risk of drug-associated QT prolongation

polymertase chain reaction (RT-PCR) confirmed COVID-19 diagnosis at Istanbul Bahcelievler State Hospital between 1-30 April, 2020. This was a single-center, retrospective, observational study. The study was performed in compliance with the Declaration of Helsinki and ethic approval was obtained from both, Ministry of Health and the local ethical committee of the hospital.

Medical records were reviewed to obtain epidemiologic characteristics of study population, baseline and control ECGs, comorbidities and laboratory findings.

According to recorded treatment orders, on day 1 all of the patients had been orally treated with $400 \mathrm{mg}$ HQ twice a day and $500 \mathrm{mg} \mathrm{AZ}$ once a day and followed by $200 \mathrm{mg}$ HQ twice a day and $250 \mathrm{mg}$ AZ once daily for four days. None of the patients experienced early discontinuation of treatment or dosage reduction due to an adverse event. All patients continued treatment for 5 days.

For each patient we calculated Tisdale score (Tab. 1) (12) which evaluates the risk for drug-associated QT corrected (QTc) prolongation.

Baseline ECGs which were recorded before the treatment could be obtained as a hard copy by examining the patient files. End points of interest were difference between pre-treatment and posttreatment values of Tp-e interval, QT interval, QTc interval $\mathrm{Tp}-\mathrm{e} / \mathrm{QT}$ ratio and $\mathrm{Tp}-\mathrm{e} / \mathrm{QTc}$ ratio.

We excluded patients with any bundle branch block, atrioventricular block, pre-excitation syndromes, atrial fibrillation and pacemaker rhythm from the study. All patients were in sinus rhythm.

All of the ECGs had been recorded using Mortara ELI-250 C ECG Machine and all ECG data were scanned and transferred to a personal computer and used for $\mathrm{x} 400 \%$ magnification with AdobePhotoshop software (Adobe Systems, Inc. San Jose, CA, USA). ECGs were examined manually by 2 different cardiologists to decrease error. After Tp-e interval, QT interval and QTc interval were measured manually, Tp-e/QT and Tp-e/QTc ratios were calculated from these measurements. The Tp-e interval was measured from the peak of the $\mathrm{T}$ wave to the end of the $\mathrm{T}$ wave, defined as the intersection of the tangent to the downslope of the $\mathrm{T}$ wave with the isoelectric line. QT interval was measured from beginning of QRS complex to end of T wave, and corrected for heart rate, using Bazett's Formula (13-15).
As far as we could access from patient files, we noticed that after the start of treatment, the day in which the control ECG was recorded differed among patients. We noted the number of days passed from the first dose through the control ECG.

\section{Statistical analysis}

Normality was tested with Shapiro-Wilk test. Normally distributed data were presented as mean \pm standard deviation and comparisons between two independent groups were performed with indepdent samples t-test. Non-normal variables were presented with median (min-max) or median (Q1-Q3) values. Comparisons for non-normal variables were performed with Mann-Whitney test for two independent groups, with Kruskal-Wallis test for more than two independent groups and with Wilcoxon test for two dependent groups. Categorical variables were presented with frequency and percentage values. Spearman and Pearson correlation coefficients were used to investigate the relationhips between the variables. Statistical significance level was taken to be $\alpha=0.05$. All statistical analyses were performed using IBM SPSS Statistics version 22.0.

\section{Results}

Among 81 patients receiving hydroxychloroquine and concomitant azithromycin; 51 patients $(63 \%)$ were male, and the mean (SD) age was 57.4 (14.8) years. Comorbid conditions were hypertension (in 31 patients [38.3\%]), diabetes mellitus (in 21 patients [25.9\%]), coronary artery disease (in 8 patients [9.9\%]), heart failure (in 3 patients [3.7 \%]) (Tab. 2). Median (min-max) Tisdale score at treatment initiation was 7 (6-14) points. Laboratory parameters of patients are listed in Table 3.

The overall baseline mean \pm SD QTc was $410.95 \pm 19.91$ (min-max: 364-455) milliseconds (ms). None of the patients who received $\mathrm{HQ} / \mathrm{AZ}$ combination therapy developed prolonged QTc of 500 milliseconds or more. Similarly, changes in QTc values were lower than $60 \mathrm{~ms}$ (maximum $57 \mathrm{~ms}$ ). A total of 48 patients $(59.3 \%$ )

Tab. 2. Basic demographic and clinical characteristics of patients.

\begin{tabular}{|c|c|c|}
\hline & & $\begin{array}{c}\text { Study } \\
\text { population } \\
\mathrm{n}: 81\end{array}$ \\
\hline \multicolumn{2}{|c|}{ Age(years), mean \pm SD } & $57.4 \pm 14.8$ \\
\hline \multicolumn{2}{|c|}{ Sex (male), n (\%) } & $51(63)$ \\
\hline \multicolumn{2}{|c|}{ Diabetes mellitus, n (\%) } & $21(25.9)$ \\
\hline \multicolumn{2}{|c|}{ Hypertension, n (\%) } & $31(38.3)$ \\
\hline \multicolumn{2}{|c|}{ Coronary artery disease, $\mathrm{n}(\%)$} & $8(9.9)$ \\
\hline \multicolumn{2}{|c|}{ Heart failure, $\mathrm{n}(\%)$} & $3(3.7)$ \\
\hline \multicolumn{2}{|c|}{ Fever, n $(\%)$} & $53(65.4)$ \\
\hline \multicolumn{2}{|c|}{ Tachycardia, n (\%) } & $13(16)$ \\
\hline \multicolumn{2}{|c|}{ Tachypnea, n (\%) } & $25(30.9)$ \\
\hline \multicolumn{2}{|c|}{ Time between 2 ECG (days), median (min-max) } & $3(1-4)$ \\
\hline \multicolumn{2}{|c|}{ Tisdale score at treatment initiation, median (min-max) } & $7(6-14)$ \\
\hline \multicolumn{2}{|c|}{ Acute cardiac injury, $n(\%)$} & $10(12.3)$ \\
\hline \multicolumn{2}{|c|}{$\geq 2$ Systemic Inflammatory Response Syndrome criteria, n(\%) } & $31(38.3)$ \\
\hline \multirow{3}{*}{ Risk level } & Low, $\mathrm{n}(\%)$ & $21(25.9)$ \\
\hline & Medium, n (\%) & $49(60.5)$ \\
\hline & High, n $(\%)$ & $11(13.6)$ \\
\hline
\end{tabular}

Data are given as mean \pm standard deviation or $\mathrm{n}(\%)$ or Median (min-max)) 
had QTc prolongation (median [min-max]: $10 \mathrm{~ms}$ [10-57]). However, the QTc prolongation was not statistically significant (Tab. 4).

Time from the first dose through the day in which the control ECG was recorded was median (min-max) 3 (1-4) days. After the treatment had started, 16 patients $(19.7 \%)$ had the control ECG on day 1,20 patients $(24.7 \%)$ had the control ECG on day 2, 36 patients $(44.4 \%)$ had the control ECG on day 3 and 9 patients $(11.1 \%)$ had the control ECG on day 4.

PTp-e interval increased from median [IQR]:75 [8.5] miliseconds to median [IQR]: 79 [10] miliseconds $(p<0.001)$, Tp-e/ QT ratio increased from median [IQR]: 0.205 [0.022] to median [IQR]: 0.206 [0.024] $(\mathrm{p}=0.002)$ and Tp-e/QTc ratio increased from

Tab. 3. Laboratory parameters of patients.

\begin{tabular}{|c|c|}
\hline & Pre-Treatment \\
\hline Glucose $(\mathrm{mg} / \mathrm{dL})$, median (min-max) & $102(67-308)$ \\
\hline Urea $(\mathrm{mg} / \mathrm{dL}), \operatorname{median}(\min -\max )$ & $29(7-93)$ \\
\hline Creatinine $(\mathrm{mg} / \mathrm{dL})$, median $(\min -\max )$ & $0.75(0.46-1.60)$ \\
\hline AST (IU/L), median (min-max) & $28(7-105)$ \\
\hline ALT (IU/L), median (min-max) & $27(3-120)$ \\
\hline Sodium $(\mathrm{mmol} / \mathrm{L})$, median $(\min -\max )$ & $138(127-149)$ \\
\hline Potassium $(\mathrm{mmol} / \mathrm{L})$, mean $\pm \mathrm{SD}$ & $4.08 \pm 0.41$ \\
\hline Calcium $(\mathrm{mg} / \mathrm{dL})$, mean $\pm \mathrm{SD}$ & $8.57 \pm 0.48$ \\
\hline Magnesium (mg/dL), mean $\pm \mathrm{SD}$ & $1.98 \pm 0.21$ \\
\hline CK (IU/L), median (min-max) & $59(8-1937)$ \\
\hline CK-MB (IU/L), median (min-max) & $15(5-93)$ \\
\hline Troponin I (pg/ml), median (min-max) & $5.10(1.10-371)$ \\
\hline LDH (IU/L), median (min-max) & $2878(150-1319)$ \\
\hline $\mathrm{CRP}(\mathrm{mg} / \mathrm{L})$, median $(\min -\max )$ & $80(1.1-487)$ \\
\hline D-DIMER ( $\mu \mathrm{g}$ FEU/mL), median (min-max) & $0.46(0.01-36)$ \\
\hline Ferritin $(\mathrm{ng} / \mathrm{ml})$, median $(\min -\max )$ & $208(9.3-1500)$ \\
\hline Albumin $(\mathrm{g} / \mathrm{L})$, median $(\min -\max )$ & $36.6(25.6-45.1)$ \\
\hline WBC $\left(\right.$ x $\left.10^{9} / \mathrm{L}\right)$, median $(\min -\max )$ & $5.93(2.39-16.01)$ \\
\hline Neutrophil (x 10/L), median (min-max) & $3.90(1.14-10.08)$ \\
\hline Lymphocyte (x $\left.10^{9} / \mathrm{L}\right)$, median (min-max) & $1.17(0.53-3.94)$ \\
\hline Platelet $\left(\right.$ x $\left.10^{9} / \mathrm{L}\right)$, median $(\min -\max )$ & $242(55-583)$ \\
\hline
\end{tabular}

Tab. 4. Electrocardiographic parameters of patients.

\begin{tabular}{lccc}
\hline & Pre-Treatment & Post-Treatment & $\mathrm{p}$ \\
\hline Tp-e (ms), median (IQR) & $75(8.5)$ & $79(10)$ & $<0.001$ \\
QT, median (IQR) & $370(40)$ & $390(36)$ & $<0.001$ \\
QTc, mean \pm SD & $410.95 \pm 19.91$ & $413.06 \pm 22.41$ & 0.257 \\
Tp-e/QT, median (IQR) & $0.205(0.022)$ & $0.206(0.024)$ & 0.002 \\
Tp-e/QTc, median (IQR) & $0.18(0.02)$ & $0.19(0.02)$ & $<0.001$ \\
\hline
\end{tabular}

ms: milisecond, SD: standard deviation QTc: QT corrected

Data are given as mean \pm standard deviation or $\mathrm{n}(\%)$ or Median (interquartile range).

Tab. 5. Comparison of risk levels according to Tisdale score with percentage change of values of ventricular repolarization indexes.

\begin{tabular}{lcccc}
\hline & $\begin{array}{c}\text { Low } \\
\text { Risk Level }\end{array}$ & $\begin{array}{c}\text { Medium } \\
\text { Risk Level }\end{array}$ & $\begin{array}{c}\text { High } \\
\text { Risk Level }\end{array}$ & $\mathrm{p}$ \\
\hline Tp-e (ms), median (IQR) & $0.05(0.04)$ & $0.05(0.04)$ & $0.05(0.04)$ & 0.950 \\
QT, median (IQR) & $0.03(0.01)$ & $0.03(0.05)$ & $0.03(0.05)$ & 0.646 \\
QTc, median (IQR) & $0.01(0.02)$ & $0.01(0.07)$ & $0.00(0.03)$ & 0.476 \\
Tp-e/QT, median (IQR) & $0.01(0.04)$ & $0.01(0.04)$ & $0.01(0.08)$ & 0.731 \\
Tpe/QTc, median (IQR) & $0.04(0.04)$ & $0.04(0.06)$ & $0.04(0.10)$ & 0.905 \\
\hline
\end{tabular}

Data given as median (interquartile range). median [IQR]: 0.18 [0.02] to median [IQR]: 0.19 [0.02] $(\mathrm{p}<0.001)$, although change in QTc prolongation was statistically unsignificant (from mean \pm SD: $410.95 \pm 19.91$ miliseconds to mean \pm SD: $413.06 \pm 22.41$ miliseconds, $\mathrm{p}=0.257$ ) (Tab. 4). Percantage changes were calculated for the ventricular repolarization indexes according to the following formula: [(posttreatment value-pretreatment value)/pretreatment value].

There was no statistically significant difference between risk levels (low, medium, high) of the patients grouped according to Tisdale score at admission, in terms of percentage changes in the ventricular repolarization indexes (Tab. 5).

Correlation analysis showed that there was a positive correlation between QTc percentage change and D-dimer $(r=0.257, p$ $=0.021$ ). Also there was a negative correlation between $\mathrm{Tpe} / \mathrm{QTc}$ percentage change and D-dimer $(r=-0.242, \mathrm{p}=0.029)$.

\section{Discussion}

In this study we found that Tp-e interval may increase even if there is not an increase in QTc interval in patients treated with HQ/AZ combination for COVID-19.

In the direction of the guidelines prepared by the Republic of Turkey, Ministry of Health (16), if the physician considers that it is neccessary, even in early stages of the disease, $\mathrm{HQ} / \mathrm{AZ}$ combination may be advised for patients who do not need to be followed at intensive care unit.

Gautret et al suggested using HQ/AZ combiantion before respiratory distress syndrome develops (8), but some subsequent studies have failed to replicate these findings $(17,18)$.

Data from a recent Chinese report showed that $16.7 \%$ of hospitalized COVID-19 patients and $44.4 \%$ of those in intensive care units had arrhythmias (19). Using QT-prolonging medications may further increase the risk of arrhythmia. HQ/AZ combination may be dangerous leading to severe cardiac disorders Both drugs have pro-arrhythmic effects. Also, chloroquine and hydroxychloroquine can cause myocardial toxicity directly.

Although hydroxychloroquine and azithromycin are generally well-tolerated medications, both can cause torsade de pointes (TdP) and corrected QT (QTc) prolongation even at therapeutic doses $(20,21)$. However, the association between QT prolongation and risk of TdP is complex. The risk of TdP is not a linear function of basal QTc or amount of QTc interval prolongation and not all patients with prolonged QTc develop TdP. Using multiple QT prolonging drugs together could increase this risk (22). Besides TdP, atrioventricular block and bundle branch block have been also reported during both acute and chronic administration of QT prolonging drugs.

Hydroxychloroquine is reported to be safe enough for patients with autoimmune disorders. However, these patients are clinically different from the patients with COVID-19 who are critically ill and receiving multiple QTc prolonging medications with extended half-lives, which augment cardiotoxic risks. Hence, some patients may 
817-821

be at elevated clinical risk for drug-induced ventricular arrhythmia. Many factors including female sex, structural heart disease, congenital long-QT syndromes, electrolyte disturbances, hepatic/ renal failure, hypoglycemia, diabetes and using concomitant QT prolonging drugs may increase risk of TdP (23).

Although previous studies have shown that QT prolongation due to $\mathrm{AZ}$ is higher in the elderly and males (24), no correlation was found between ECG findings and age or gender in our study.

Chorin et al reported changes in QT interval of 84 patients who were treated with hydroxychloroquine and azithromycin for COVID-19. None of the patients experienced cardiac arrest during the study, but $11 \%$ had QT interval prolongation (24).

Bessière $\mathrm{F}$ et al reported in their study that most patients (37 [93\%]) treated for COVID-19 showed an increase in QTc after the administration of antiviral therapy. Prolonged QTc was observed in 14 patients $(36 \%)(10$ with $\Delta$ QTc $>60$ milliseconds and 7 with QTc $\geq 500$ milliseconds) after a duration of antiviral treatment of 2 to 5 days. No ventricular arrhythmia, including torsades de pointes, was recorded (25). On the other hand, in our study none of the patients had neither a value of QTc interval more than $500 \mathrm{~ms}$ or a change in QTc over $60 \mathrm{~ms}$ and none of them experienced cardiac arrest. In our study, prolongation of QTc interval was not statistically significant.

Furthermore, Guo et al reported that 19-33\% of hospitalized patients with COVID-19 have concurrent cardiac injury (26) which can further increase the risk of arrhythmias. Underlying mechanisms may be severe systemic inflammatory responses, direct injury from the severe acute respiratory syndrome or microvascular damage due to microthrombi (27). In our study group, 10 patients $(12.3 \%)$ had acute cardiac injury at admission to the hospital.

Myocardial repolarization can be assessed using various methods. Tisdale score and additional ventricular repolarization indexes other than QTc may be useful to better assess the risk of arrhythmia. Tp-e interval and Tp-e/QT ratio have emerged as novel ECG markers for increased dispersion of ventricular repolarization (13, 14, 28). Tp-e interval on ECG has been reported to be a ventricular repolarization index $(29,30)$ and associated with ventricular arrhythmogenesis and sudden cardiac death $(15,31,32)$. Even if Tp-e interval is affected by heart rate (33), Tp-e/QTc ratio which is another ventricular arrhythmia index (32) is not affected by changes in heart rate $(33,34)$.

During treatment of COVID-19, Canadian guideline advises to record the control ECG in 48 hours (35), in our study median time from start of the treatment through control ECG was 3 days.

Performing daily serial ECGs for QTc monitoring may increase exposures and personal protective equipment (PPE) use. Because of exposure risk to the virus, healthcare workers may hesitate to record control ECG daily. Due to this situation, ECG follow-up must not be neglected and even alternative rhythm monitoring methods such as ECG telemetry should be used. It should not be forgotten that the risk of arrhythmia is high, especially when using combined QT-prolonging drugs.

Although the benefit of HQ/AZ combination in COVID-19 treatment is controversary, it seems that these drugs will be continued to be used until more effective drugs or vaccines are developed. HQ/AZ combination may cause cardiac arrhythmia and it should be kept in mind that QTc monitoring may not be sufficient to evaluate arrhythmia risk. Hence, ventricular repolarization indexes may be an alternative option for this purpose.

As far as we know, there is no other study in the literature concerning the association between COVID-19 and Tp-e interval, Tp-e/QT ratio, Tp-e/QTc ratio. We have shown for the first time that in patients with COVID-19, without a statistically significant prolongation in QTc, the Tp-e interval may be prolonged and the Tp-e/QT, Tp-e/QTc ratios may increase.

Our results are in consistence with the results of the studies evaluating the effect of smoking (36) and subclinical hypothyroidism (37) on ventricular repolarization indexes. Similar to our study, these studies have shown that without a concomitant QTc prolongation Tp-e interval and Tp-e/QTc ratio may increase.

\section{Conclusion}

It is necessary to investigate whether this statistically significant result is only an ECG finding or whether it is also associated with arrhythmias. Post-discharge follow-up and large-scale prospective studies are needed to investigate the arrhythmia predictive values of Tp-e interval, Tp-e/QT ratio and Tpe/QTc ratio in terms of arrhythmia occurence in patients treated for COVID-19.

\section{Limitations}

Our study has some limitations. This study had relatively small sample size and is a single-center study. A prior report revealed that the maximal change in the QT interval among patients treated with HQ/AZ combination for COVID-19 occurred between days 3 and 4 (24). In our study, we could not access daily recorded ECGs and among present ECG records, the days when the control ECGs were recorded were variable, hence we do not know at which day after treatment the increase in ventricular repolarization indexes started. Besides, since the patients included in the study were not followed up by telemetry, we do not know whether the detected increase in ventricular repolarization indexes on ECG is related to a silent arrhythmia. Lastly, since the terminal elimination half-life of hydroxychloroquine is more than 40 days, patients may have a rhythm disorder after discharge but long term follow up results of our study population are not yet available.

\section{References}

1. Binti Hamzah FA, Lau C, Nazri $\mathbf{H}$ et al. CoronaTracker: world-wide COVID-19 outbreak data analysis and prediction. Bull World Health Organ. DOI: http://dx.doi.org/10.2471/BLT.20.255695.

2. Dong E, Du H, Gardner L. An interactive web-based dashboard to track COVID-19 in real time. Lancet Infect Dis 2020. DOI: 10.1016/S1473-3099 (20)30120-1.

3. Jin Y, Yang H, Ji W, Wu W, Chen S, Zhang W, Duan G. Virology, Epidemiology, Pathogenesis, and Control of COVID-19. Viruses 2020; 12 (4). pii: E372. doi: 10.3390/v12040372.

4. Liu J, Cao R, Xu M et al. Hydroxychloroquine, a less toxic derivative of chloroquine, is effective in inhibiting SARS-CoV-2 infection in vitro. Cell Discov 6, 16 (2020). https://doi.org/10.1038/s41421-020-0156-0 
5. Retallack H, Di LE, Arias C, Knopp KA, Laurie MT, SandovalEspinosa $\mathbf{C}$ et al. Zika virus cell tropism in the developing human brain and inhibition by azithromycin. Proc Natl Acad Sci USA 2016; 113 (50): 14408-14413.

6. Bosseboeuf E, Aubry M, Nhan T, de Pina JJ, Rolain JM, Raoult D et al. Azithromycin inhibits the replication of Zika virus. J Antivir Antiretrovir 2018; 10 (1): 6-11.

7. Li C, Zu S, Deng YQ, Li D, Parvatiyar K, Quanquin N et al. Azithromycin protects against $Z i k a$ virus infection by upregulating virus-induced type I and III interferon responses. Antimicrob Agents Chemother 2019. https://doi. org/10.1128/AAC.00394-19.

8. Gautret P, Lagier JC, Parola P, Meddeb L, Mailhe M, Doudier B, Courjon J, Giordanengo V, Vieira VE, Dupont HT. Hydroxychloroquine and azithromycin as a treatment of COVID-19: results of an open-label non-randomized clinical trial. Internat J Antimicrob Agents 2020; 105949.

9. Mercuro NJ, Yen CF, Shim DJ et al. Risk of QT Interval Prolongation Associated With Use of Hydroxychloroquine With or Without Concomitant Azithromycin Among Hospitalized Patients Testing Positive for Coronavirus Disease 2019 (COVID-19). JAMA Cardiol 2020. DOI: 10.1001/ jamacardio.2020.1834.

10. Simpson TF, Kovacs RJ, Stecker EC. Ventricular arrhythmia risk due to hydroxychloroquine-azithromycin treatment for COVID-19. Cardiol Mag 2020.

11. McCreary EK, Pogue JM. COVID-19 Treatment: A Review of Early and Emerging Options. Open Forum Infectious Diseases. Accepted manuscript.

12. Tisdale JE, Jayes HA, Kingery JR et al. Development and validation of a risk score to predict QT interval prolongation in hospitalized patients. Circ Cardiovasc Qual. Outcomes 2013; 6 (4): 479-487. DOI: 10.1161/ CIRCOUTCOMES.113.000152.

13. Acar GR, Akkoyun M, Nacar AB, Dirnak I, Yildirim Cetin G, Nur Yildirim $\mathrm{M}$ et al. Evaluation of Tp-e interval and Tp-e/QT ratio in patients with rheumatoid arthritis. Turk Kardiyol Dern Ars 2014; 42: 29-34.

14. Yayla C, Bilgin M, Akboga MK, Gayretli Yayla K, Canpolat U, Dinc Asarcikli L et al. Evaluation of Tp-E interval and Tp-E/QT ratio in patients with aortic stenosis. Ann Noninvasive Electrocardiol 2016; 21: 287-293.

15. Zhao X, Xie Z, Chu Y, Yang L, Xu W, Yang X et al. Association between $\mathrm{Tp}-\mathrm{e} / \mathrm{QT}$ ratio and prognosis in patients undergoing primary percutaneous coronary intervention for ST-segment elevation myocardial infarction. Clin Cardiol 2012; 35: 559-564.

16. COVID-18 Guideline prepared by the Republic of Turkey, Ministry of Health, available at https://covid19bilgi.saglik.gov.tr/depo/rehberler/COVID-19_Rehberi.pdf?type=file.

17. Molina JM, Delaugerre C, Le Goff J et al. No evidence of rapid antiviral clearance or clinical benefit with the combination of hydroxychloroquine and azithromycin in patients with severe COVID-19 infection. Med Mal Infect. DOI:10.1016/j.medmal.2020.03.006.

18. Mehra MR, Desai SS, Ruschitzka F, Patel AN. Hydroxychloroquine or chloroquine with or without a macrolide for treatment of COVID-19: a multinational registry analysis. Lancet 2020; S0140-6736(20)31180-6. DOI: 10.1016/S0140-6736(20)31180-6.

19. Wang D, Hu B, Hu C, Zhu F, Liu X, Zhang J, Wang B, Xiang H, Cheng Z, Xiong Y, Zhao Y, Li Y, Wang X, Peng Z. Clinical characteristics of 138 hospitalized patients with 2019 novel coronavirus-infected pneumonia in Wuhan, China. JAMA 2020; 323: 1061-1069

20. World Health Organization. The cardiotoxicity of antimalarials: Malaria Policy Advisory Committee Meeting. Published March 24, 2017. Accessed April 21, 2020.
21. RayWA, Murray KT, Hall K, Arbogast PG, Stein CM. Azithromycin and the risk of cardiovascular death. N Engl J Med 2012; 366 (20): 18811890. DOI: 10.1056/NEJMoa1003833.

22. Rock EP, Finkle J, Fingert HJ et al. Assessing proarrhythmic potential of drugs when optimal studies are infeasible. Am Heart J 2009; 157 (5): 827-836.

23. The Cardiotoxicity of Antimalarials. World Health Organization- Malaria Policy Advisory Committee Meeting. 22 Mar, 2017.

24. Chorin E, Dai M, Shulman $\mathbf{E}$ et al. The QT interval in patients with COVID-19 treated with hydroxychloroquine and azithromycin. Nat Med (2020). https://doi.org/10.1038/s41591-020-0888-2,

25. Bessière F, Roccia $\mathbf{H}$, Delinière $\mathbf{A}$ et al. Assessment of QT Intervals in a Case Series of Patients With Coronavirus Disease 2019 (COVID-19) Infection Treated With Hydroxychloroquine Alone or in Combination With Azithromycin in an Intensive Care Unit. JAMA Cardiol 2020. DOI: 10.1001/ jamacardio.2020.1787.

26. Guo T, Fan Y, Chen M, Wu X, Zhang L, He T, Wang H, Wan J, Wang X, Lu Z. Cardiovascular implications of fatal outcomes of patients with coronavirus disease 2019 (COVID-19). JAMA Cardiol 2020. DOI: 10.1001/jamacardio.2020.1017.

27. Li Y, Wang M, Zhou Y et al. Acute cerebrovascular disease following COVID-19: a single center, retrospective, observational study. http://dx.doi. org/10.2139/ssrn.3550025.

28. Castro-Torres Y, Carmona-Puerta R, Katholi RE. Ventricular repolarization markers for predicting malignant arrhythmias in clinical practice. World J Clin Cases 2015; 3: 705-720.

29. Antzelevitch C, Shimizu W, Yan GX, Sicouri S. Cellular basis for QT dispersion. J Electrocardiol 1998; 30 Suppl: 168-175.

30. Antzelevitch C. T peak-Tend interval as an index of transmural dispersion of repolarization. Eur J Clin Invest 2001; 31: 555-557.

31. Castro Hevia J, Antzelevitch C, Tornes Barzaga F, Dorantes Sanchez M, Dorticos Balea F, Zayas Molina R et al. Tpeak-Tend and Tpeak-Tend dispersion as risk factors for ventricular tachycardia/ventricular fibrillation in patients with the Brugada syndrome. J Am Coll Cardiol 2006; 47: 1828-1834.

32. Sicouri S, Antzelevitch C. A subpopulation of cells with unique electrophysiological properties in the deep subepicardium of the canine ventricle. The M cell. Circ Res 1991; 68: 1729-1741.

33. Gupta P, Patel C, Patel H, Narayanaswamy S, Malhotra B, Green JT et al. T pe/QT ratio as an index of arrhythmogenesis. J Electrocardiol 2008; 41: 567-574.

34. Watanabe N, Kobayashi Y, Tanno K, Miyoshi F, Asano T, Kawamura $\mathbf{M}$ et al. Transmural dispersion of repolarization and ventricular tachyarrhythmias. J Electrocardiol 2004; 37: 191-200.

35. Sapp JL, Alqarawi W, MacIntyre CJ et al. Guidance on Minimizing Risk of Drug-Induced Ventricular Arrhythmia During Treatment of COVID-19: A Statement from the Canadian Heart Rhythm Society. Can J Cardiol 2020, pii: S0828-282X(20)30325-1. DOI: 10.1016/j.cjca.2020.04.003.

36. Kayali S, Demir F. The effects of cigarette smoking on ventricular repolarization in adolescents. Einstein (Sao Paulo) 2017; 15 (3): 251-255.

37. Gürdal A, Eroğlu H, Helvaci F et al. Evaluation of Tp-e interval, Tp-e/ QT ratio and Tp-e/QTc ratio in patients with subclinical hypothyroidism Ther Adv Endocrinol Metab 2017; 8 (3): 25-32. DOI: 10.1177/2042018816684423. 